\title{
The absence of pyruvate kinase is essential in genome streamlining of Marine Group IIb archaea
}

\author{
H. LIU ${ }^{1,2} ;$ B. XU ${ }^{1} ;$ L. QIN $^{2} ;$ C. ZHANG ${ }^{1 *}$
}

${ }^{1}$ Shenzhen Key Laboratory of Marine Archaea Geo-Omics, Southern University of Science and Technology, Shenzhen, China (1iuhd@sustech.edu.cn;

*correspondence: zhangcl@sustech.edu.cn )

${ }^{2}$ CAS Key Laboratory of Crust-Mantle Materials and

Environments, University of Science and Technology of China, Hefei, China

MGIIa and MGIIb are two major groups of marine planktonic Euryarchaeota; the former predominate in eutrophic marine environments with relatively large genomes (2.1-2.5 Mbp) and the latter in oligotrophic marine environments with the majority of them containing smaller genomes (1.5-1.9 Mbp) that apparently resulting from genome streamlining. We analyzed high quality metagenome-assembled genomes (MAGs) of MGII from public datasets and linked the genome streamlining of MGIIb to the absence of pyruvate kinase that resulted in incomplete glycolytic pathway producing less ATP and pyruvate. Correspondingly, they lost genes related to saccharides degradation, such as glycoside hydrolases. Many other genes are lost to reduce the consumption of ATP, including sodium ABC-type transport proteins and archaeal flagellar proteins, as well as genes non-essential for metabolism and proliferation, such as rod shape-determining protein MreB and related proteins. On the other hand, the genomestreamlined MGIIb gained genes related to metabolism of $\mathrm{N}$ containing organic compounds, such as D-amino acid oxidase and general L-amino acid transport system proteins. These results demonstrate that the absence of pyruvate kinase in genome-streamlined MGIIb likely forced them to prefer Ncontaining organic compounds and recalcitrant dissolved organic matter metabolism, which facilitated their adaption to oligotrophic marine environments that are depleted in fixed nitrogen. 